\title{
ELECTRICAL AND ESR STUDIES OF GaN LAYERS GROWN BY METAL ORGANIC CHEMICAL VAPOUR DEPOSITION
}

\author{
B. Suchanek ${ }^{a}$, M. Palczewska ${ }^{b}$, K. Pakula ${ }^{a}$, J. Baranowski ${ }^{a}$ \\ AND M. KAMIŃSKA ${ }^{a}$ \\ ${ }^{a}$ Institute of Experimental Physics, Warsaw University \\ Hoża 69, 00-681 Warsaw, Poland \\ ${ }^{6}$ Institute of Technology of Electronic Materials \\ Wólczyńska 133, 01-919 Warsaw, Poland
}

Electrical transport and ESR studies were performed on the state-of-the-art GaN layers grown on sapphire substrate using metal organic chemical vapour deposition technique. For undoped samples electron concentration below $2 \times 10^{17} \mathrm{~cm}^{-1}$ and mobility up to $500 \mathrm{~cm}^{2} /(\mathrm{V} \mathrm{s})$ were achieved whereas hole concentration up to $7 \times 10^{17} \mathrm{~cm}^{-3}$ and mobility about $16 \mathrm{~cm}^{2} /(\mathrm{V}$ s) were obtained for intentionally $\mathrm{Mg}$ doped samples and subsequently annealed. Temperature dependence of mobility was discussed. ESR revealed the presence of two resonance absorption lines. One of them with $g_{\perp}=1.9487$ and $g_{\|}=1.9515$, commonly observed in $n$-type $\mathrm{GaN}$ was due to shallow donor. The second ESR line was an isotropic one of $g=2.0032$ and it is discussed.

PACS numbers: 72.80.Ey, 73.61.Ey

Gallium nitride has attracted a lot of interest in the last few years as a material of great perspectives for applications in modern optoelectronic devices working in the visible and ultraviolet light range. At the same time extensive studies of GaN crystals have been undertaken but the basic knowledge about this material is still far from satisfactory. One of the major reason limiting both applications as well as better understanding is a problem in obtaining good quality crystals which technology is far more difficult from the standard one used for growth of other III-V compounds.

GaN heteroepitaxial layers studied in this paper were grown by horizontal metal organic chemical vapour deposition (MOCVD) using adopted Epigress system in the Crystal Growth Laboratory, Warsaw University. The layers were either undoped or $\mathrm{Mg}$-doped with three different concentrations each of them twice higher than the previous one and subsequently annealed at $750^{\circ} \mathrm{C}$ using rapid thermal annealing (RTA) method to activate $p$-type dopant. Sapphire substrates were applied and the growth was initiated with deposition of low temperatures GaN buffer layer. $\mathrm{TMG}$ and $\mathrm{NH}_{3}$ served as sources of $\mathrm{Ga}$ and $\mathrm{N}$ respectively and magnesium 
Mg metalorganic was introduced during growth of doped layers. Hydrogen was used as a carrier gas. The growth was performed at temperatures close to $1000^{\circ} \mathrm{C}$ with all parameters adjusted to obtain $2 \mu \mathrm{m}$ thick GaN layers.

Electrical transport measurements using Van der Pauw method were performed on $\mathrm{GaN}$ samples placed in a close cycle refrigerator and in magnetic field in the range about $250 \mathrm{mT}$. Sample temperature varied between 13 to $400 \mathrm{~K}$. For $n$-type layers ohmic contacts were prepared using metallic indium, whereas for $p$-type evaporation of nickel and gold with subsequent annealing at $500^{\circ} \mathrm{C}$ for $30 \mathrm{~s}$ was applied.
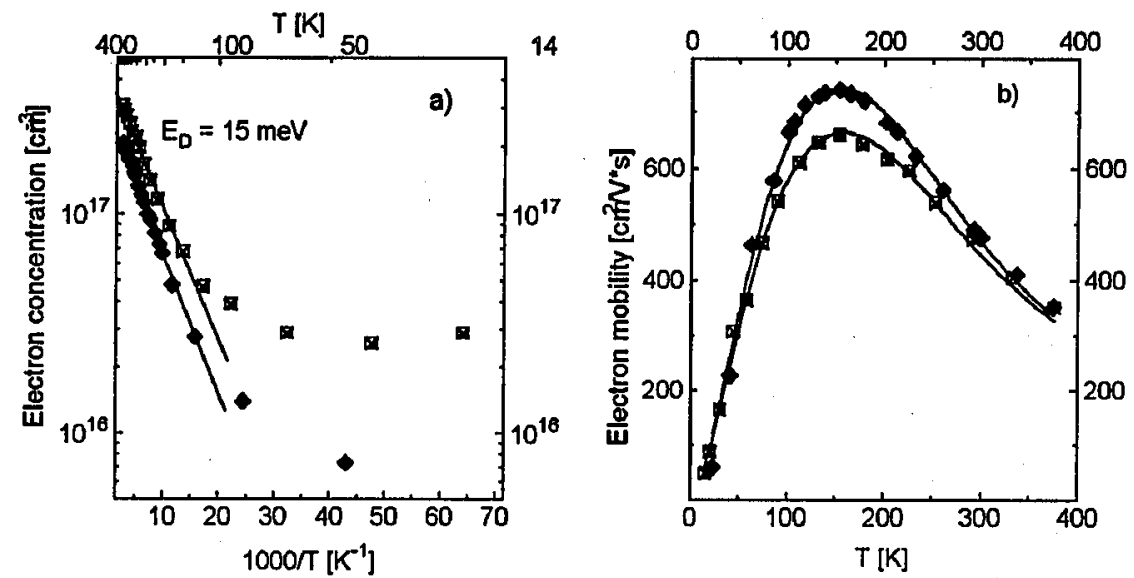

Fig. 1. Electron concentration versus inverse temperature (a) and mobility as a function temperature (b) for undoped GaN layers. Fitted curves are plotted as solid line.

In Fig. 1a electron concentration versus inverse temperature for two typical undoped GaN layers is presented. The temperature dependencies of concentrations above $200 \mathrm{~K}$ yielded a Fermi level position at $15 \mathrm{meV}$, indicating donor activation energy in the range $15-30 \mathrm{meV}$ depending on the compensation ratio $\left(N_{\mathrm{A}} / N_{\mathrm{D}}\right)$. Recent measurements of two-electron transition within donor bound exciton complex have estimated donor activation energy in GaN as $29 \mathrm{meV} \mathrm{[1].}$ This value suggested that the compensation ratio of the investigated samples is less than 0.3 [2]. Below $200 \mathrm{~K}$ electron concentration changed with lower activation energy which could be explained as a growing influence of electron transport in shallow impurity states. Electron mobility dependence versus temperature is shown in Fig. 1b. The curves had characteristic behavior for semiconductors with maximum mobility value at about $100 \mathrm{~K}$. The decrease in mobility at high temperature could be explained by lattice scattering due to the optical and acoustic mode lattice vibrations. The low temperature diminishing of mobility has been usually ascribed to impurity scattering. However, in this case impurity scattering potentials were effectively screened by high concentration of free carriers. On the other hand, the observed values of mobility ( $\left.>50 \mathrm{~cm}^{2} /(\mathrm{V} \mathrm{s})\right)$ are too high to be explained by conduction in a donor level. Therefore, an extra mechanism leading to mobility decrease at low temperature had to be taken under consider- 
ation. The possible candidate was scattering at dislocations. Such mechanism is described by $T^{-3 / 2}$ temperature dependence [3]. In Fig. 1b the calculated mobility is presented, assuming scattering at ionized impurities, dislocations and lattice vibrations. The following parameters were applied: ionized impurity concentration $10^{18} \mathrm{~cm}^{-3}$, dislocation density $10^{10} \mathrm{~cm}^{-2}$ and elastic energy $E_{1}=7.5 \mathrm{eV}$. The calculated curves fitted the experimental data quite well. However, one could not exclude other scattering mechanisms like potential fluctuation or interface influence. These mechanisms need further studies in samples with buffer layers of different thickness.

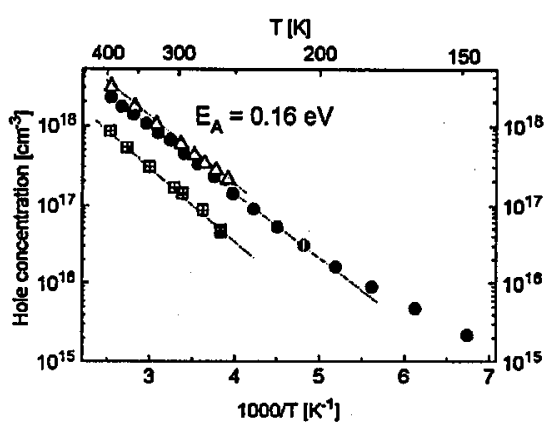

Fig. 2. Hole concentration versus inverse temperature for three GaN layers, doped with different $\mathrm{Mg}$ concentration. Fitted curves are plotted as solid lines.

In Fig. 2 hole concentration versus inverse temperature for three different $\mathrm{Mg}$ doped GaN layers is presented. The temperature dependence of concentration yielded a Fermi level position at $160 \mathrm{meV}$. This value was in agreement with the estimation of acceptor energy from effective mass theory. Therefore, high compensation ratio of the investigated $p$-type layers should be assumed.

GaN layers were examined by electron spin resonance (ESR) technique. $\mathrm{X}$-band Brucker spectrometer was used and the samples were kept in continuous flow cryostat.

Typical ESR spectra of GaN layers are shown in Fig. 3. Two resonance absorption lines were observed. One of them, single and anisotropic, with $g_{\perp}=$ 1.9487 and $g_{\|}=1.9515$ was detected only in $n$-type (undoped) samples. This line was similar to the one found by Carlos et al. [4], and ascribed to a shallow donor in $\mathrm{GaN}$. The second isotropic resonance signal with $g=2.0031$ was observed for both $\mathrm{Mg}$ doped and undoped samples. The $g$ factor of it was the same as for the signal found for GaN microcrystals grown by ammonothermal technique [5]. Recently very similar resonances were also detected by us in other nitride compounds grown under nitrogen rich conditions. Moreover, ESR signal of very close $g$ factor has been reported for BN film grown by RF diode sputtering [6]. All these observations suggested common defect or impurity as an origin of the second isotropic ESR line. Most probable candidates are oxygen, metallic vacancy or dangling bonds at the surface. 


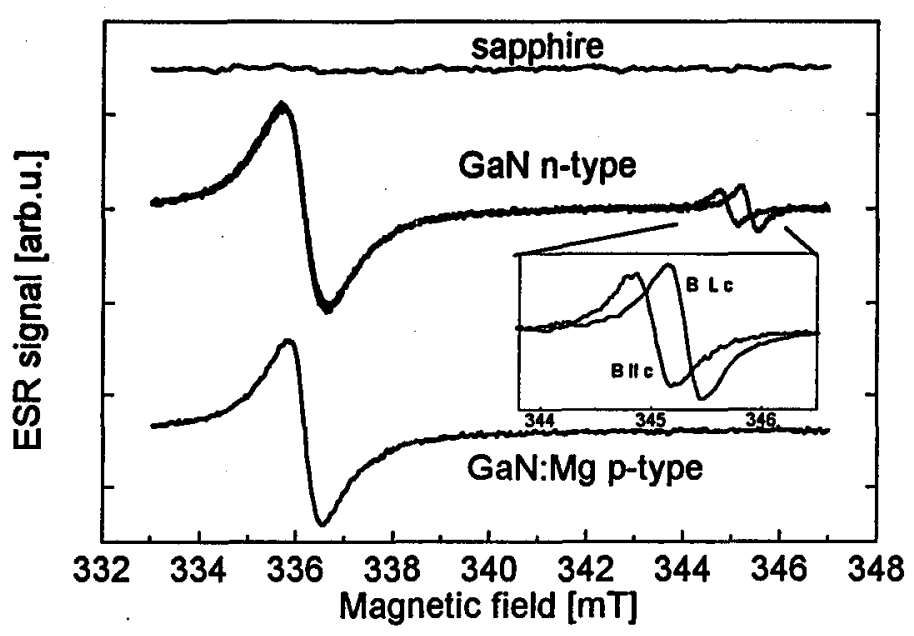

Fig. 3. ESR spectra for two layers of $\mathrm{GaN}$ and for sapphire substrate $(5 \mathrm{~K})$.

Electron mobility in the state-of-the-art GaN layers grown by MOCVD technique showed strong decrease at temperatures below $100 \mathrm{~K}$ which could not be explained by ionized impurity scattering only. Including the scattering by dislocations allowed to fit the experimental data. However, other scattering mechanisms cannot be excluded.

Two ESP absorption lines, characteristic of MOCVD layers were found. One of them was due to a shallow donor, whereas the second one most probably originated from oxygen or gallium vacancy.

This work was partly supported by the Committee for Scientific Research grant no. 8 T11 B 03310.

\section{References}

[1] A. Fiorek, J.M. Baranowski, A. Wysmołek, K. Pakuła, M. Wojdak, I. Grzegory, S. Porowski, Acta Phys. Pol. A 92, 742 (1997).

[2] P.S. Kiriejev, Fizika poluprovodnikov, Vysshaya Shkola, Moskva 1969.

[3] B. Pödör, Phys. Status Solidi 16, K167 (1966).

[4] W.E. Carlos, J.A. Freitas, M. Asif Khan, D.T. Olson, J.N. Kuznia, Phys. Rev. B 48, 17878 (1993).

[5] R. Dwiliński, J.M. Baranowski, M. Kaminiska, R. Doradziński, J. Garczyński, L. Sierzputowski, M. Palczewska, Acta Phys. Pol. A 02, 737 (1997).

[6] M. Fanciulli, T.D. Moustakas, Physica B 185, 228 (1993). 